\title{
Human Testosterone and Lactate Values from Flywheel Ergometry: Effect of Contractile Mode and Work Volume
}

\author{
John F. Caruso ${ }^{1}$, Melissa A. Urquhart ${ }^{2}$, Rachel M. Giebel ${ }^{1}$, Amanda G. Barbosa ${ }^{1}$, Christy E. Craig ${ }^{2}$,
} Melissa L. Mason ${ }^{1}$, Kayla D. Unruh ${ }^{1}$, Jake A. Borgsmiller ${ }^{1}$, and William T. Potter ${ }^{2}$

${ }^{1}$ Exercise and Sports Science Program, The University of Tulsa, Tulsa, OK; ${ }^{2}$ Department of Chemistry and Biochemistry, The University of Tulsa, Tulsa, OK

\begin{abstract}
In-flight resistive exercise workouts are performed on novel flywheel-based hardware. Designs of such workouts may be better served by measuring changes to lactate and testosterone values. To make workouts pertinent to $\mu g$ they should utilize unique features of flywheel-based hardware, such as the option to exert eccentric torque. Our study compares changes to blood lactate and testosterone concentrations ([BLa $\left.{ }^{-}\right]$, [T]) from leg press workouts that differ by contractile mode and work volume, on a flywheel ergometer. Subjects performed three workouts; two entailed two sets of concentric-eccentric (CE2) or concentric-only (CO2) actions. A third involved four sets of concentric-only actions (CO4). Workouts entailed eight-repetition sets with 90 -second rest periods. Total work (TW) was quantified per workout. [T] were assessed, both pre- and post-exercise. [BLa] ${ }^{-}$were measured pre-
\end{abstract}

Key words: Concentric; Eccentric; Total Work; Exercise; Countermeasures; Leg Presses; Mechanical Loading

Correspondence to: John Caruso

The University of Tulsa

312 Chapman Hall

800 S. Tucker Drive

Tulsa, OK 74104

Telephone: 918-631-2924

E-mail: john-caruso@utulsa.edu and at $0-$, 5-, 10-, 15-, and 20-minutes postexercise. TW was assessed with a one-way analysis of variance (ANOVA). [BLa'] and [T] were evaluated with two- and three-factor ANOVAs, respectively. Scheffe's test was our post-hoc. TW data had an inter-workout (CE2, $\mathrm{CO} 4>\mathrm{CO} 2)$ difference. [BLa $\left.{ }^{-}\right]$included a twoway interaction as $\mathrm{CO} 4$ workouts evoked higher post-exercise values. Results for [T] produced gender (men $>$ women) and time (post $>$ pre) main effects. Our results imply flywheel-based workouts with a large volume of concentric actions evoke no greater increase in [T] than workouts with only half the muscle shortening activity, despite attainment of higher TW and post-workout [BLa'].

\section{INTRODUCTION}

Long-term microgravity $(\mu g)$ exposure induces pronounced physiological changes (NASA Human Research Roadmap, 2014). Inflight muscle mass and strength losses may be best abated by an intense mechanical loading stimulus that entail frequent application of high muscle/reaction forces that characterize resistive exercise done in 1-g (Rantalainen and Klodowski, 2011). Yet if similar workouts occur in $\mu g$ they can be limited by the ability of in-flight hardware to impart sufficient mechanical loads (Garnier, 2012; Peterman et al., 2001). Flywheel-based resistive exercise hardware offers perhaps the best loading stimulus in $\mu g$, which includes the 
Advanced Resistive Exercise Device (ARED) and Flywheel Exercise Device (FWED) aboard the International Space Station (ISS) (Bentley et al., 2006; European Space Agency, 2009; International Exercise Countermeasures Working Group, 2010; Tesch and Berg, 1997).

The ARED's flywheels simulate the inertial features of weightlifting on Earth; pneumatic cylinders provide its primary source of resistance (Bentley et al., 2006). Yet a biomechanical simulation of in-flight squat repetitions on the ARED showed no amount of added shoulder resistance and offered the same level of leg muscle loading as when the exercise is done in 1$g$ (Garnier, 2012). Users compensate by adding up to $70 \%$ of their body mass to the bar, yet it was deemed insufficient to maintain leg muscle function for trips to the Moon or Mars (Garnier, 2012). In contrast, high muscle/reaction forces provided by flywheel-based hardware in $1-g$ should be more easily replicated in-flight via the FWED. Flywheels on the FWED are its main source of resistance (Tesch and Berg, 1997). Full knee extension achieved with leg presses on the FWED imparts a large axial load that should help preserve in-flight muscle mass and strength (Rantalainen and Klodowski, 2011). The resultant adaptations may evoke higher post-exercise testosterone concentrations ([T]) that enhance mass and strength outcomes.

Studies report divergent changes to [T] that vary by exercise intensity, mode, and duration (Liu et al., 2009). Exercise rapidly increases [T] but does not impose long-term changes (Liu et al., 2009), which suggest a greater exercise frequency is needed to consistently elevate [T]. $\mu g$ inhibits androgen secretion; such changes were affirmed by losses to salivary [T] in male astronauts (Strollo et al., 2005). In-flight testosterone secretion from resistive exercise may abate such losses (Ratames et al., 2005). Yet exercise suppressed [T] during bed rest, a $\mu g$ analog (Wade et al., 2005). Nonetheless, changes to [BLa ${ }^{-}$ foretell the workout's merits, as well as subsequent $[\mathrm{T}]$, and thus warrant inquiry (Caruso et al., 2010). Some believe exercise-induced increases in [T] are related to concurrent lactate production (Liu et al., 2009), while others imply the two operate independently (Kraemer et al., 1991). High blood lactate concentrations ([BLa $\left.{ }^{-}\right]$) parallel $\mathrm{H}^{+}$accrual; the latter compromises exercise performance (Caruso et al., 2010). Since similar [BLa'] occurred in $\mu g$ and 24 hours later when the same workout was replicated on Earth (Zamparo et al., 1992), perhaps $1-g$ research is a viable analog to assess in-flight exercise-induced lactate changes. High [BLa $\left.{ }^{-}\right]$may foretell similar changes to $[\mathrm{T}]$ that impact muscle mass and function (Bosco et al., 1996a; Bosco et al., 1996b).

Perhaps the merits of flywheel-based workouts are best assessed by monitoring changes to testosterone and lactate. To make workouts more pertinent to $\mu g$ they should include unique features of flywheel-based hardware such as the option to exert eccentric torque, as muscle lengthening under tension may abate mass and strength losses (Stauber, 1989; Tesch and Berg, 1997). However, historically in-flight exercise modes included large volumes of concentric activity, which did little to ameliorate muscle mass and strength losses (Friel and Vance, 2013). A FWED predecessor, a prototype flywheel ergometer, has similar features, such as a large inertial resistance and the option to exert eccentric torque (Tesch and Berg, 1997). Examination of such features may aid development of flywheel prescription optimization. Thus our study compares changes to $[\mathrm{T}]$ and $\left[\mathrm{BLa}^{-}\right]$from three leg press workouts on the prototype flywheel ergometer that differ in their use of contractile modes and the volume of work performed. In terms of identified risks for long-term spaceflight, our study offers insights for flywheel prescription optimization and the need for in-flight testosterone administration (NASA Human Research Roadmap, 2014). While our study outcomes may be used to blunt muscle mass and strength losses, the application of current result to address this risk is limited by the lack of $\mu \mathrm{g}$ simulation. We hypothesize our study will yield significant changes to $[\mathrm{T}]$ and $\left[\mathrm{BLa}{ }^{-}\right]$over time.

\section{MATERIALS AND METHODS}

A university-based institutional review board approved study procedures. Healthy college-age subjects (17 men, 18 women) gave informed written consent. Male subject characteristics (mean \pm sem) were as follows: height $183.9 \pm 2.0$ $\mathrm{cm}$, mass $97.3 \pm 5.3 \mathrm{~kg}$, body fat percentage 17.6 $\pm 1.6 \%$. Characteristics of our female subjects 
were: height $165.6 \pm 1.4 \mathrm{~cm}$, mass $67.9 \pm 2.6 \mathrm{~kg}$, body fat percentage $27.4 \pm 1.6$. None had prior experience with the prototype ergometer. For our study one workout entailed two sets of concentriconly repetitions $(\mathrm{CO} 2)$, while another involved two sets that included both concentric and eccentric actions (CE2). A third workout entailed four sets of concentric-only repetitions (CO4). Workouts were comprised of eight-repetition sets. Based on prior results (Caruso et al., 2010), CE2 and $\mathrm{CO} 4$ should entail similar volumes of work, and each should be twice that of $\mathrm{CO} 2$. Figure 1 illustrates the prototype ergometer.

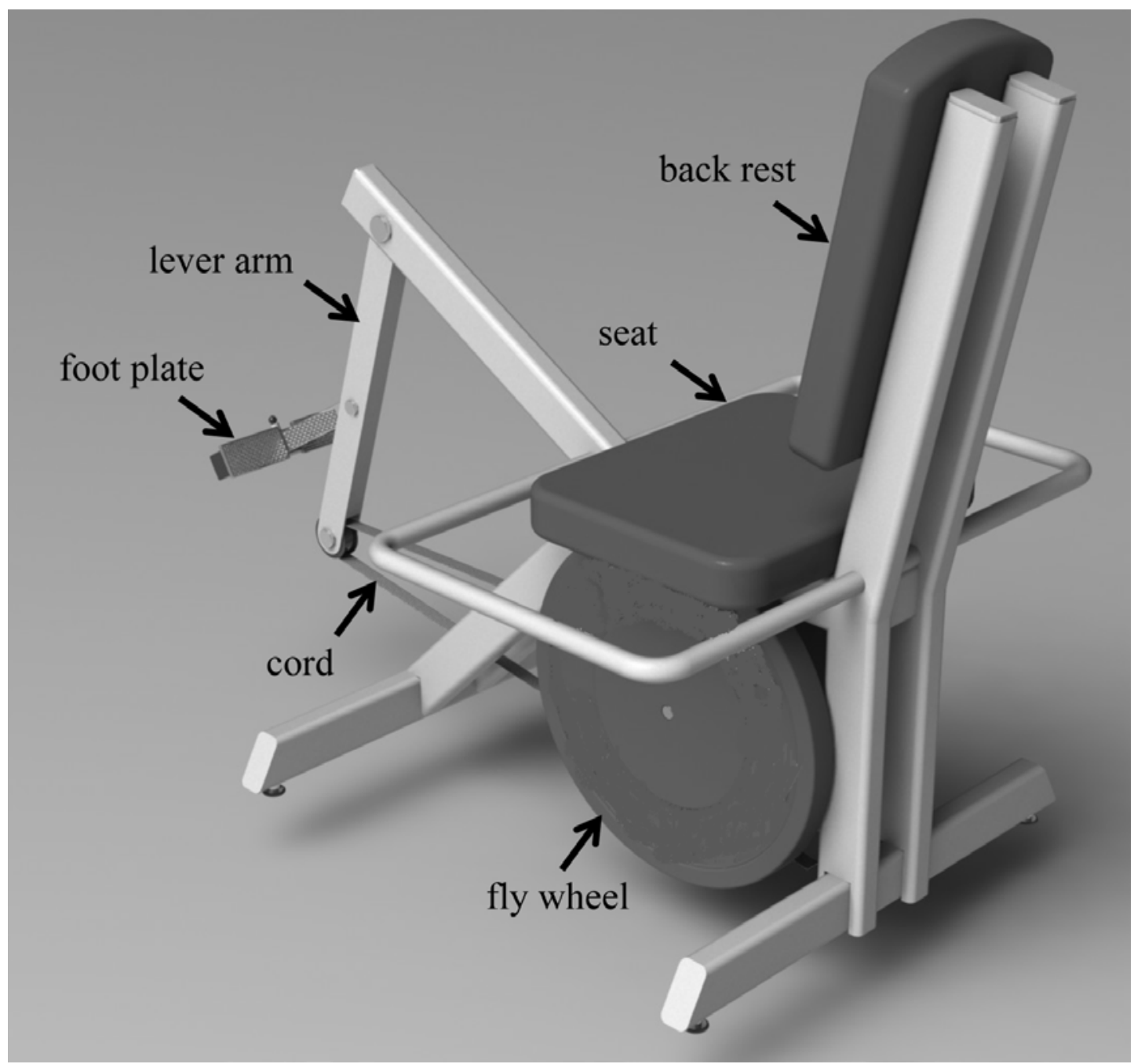

Figure 1. Illustration of the current study prototype flywheel ergometer.

Ankle, knee, and hip joint movements during leg press repetitions on the prototype ergometer mimic those of the FWED (European Space Agency, 2009; International Exercise Countermeasures Working Group, 2010). The flywheels' radii $(23 \mathrm{~cm})$ dictate the ergometer's resistance. Forces are exerted against a footplate mounted onto a lever arm that moves like a pendulum. As concentric torque overcomes the resistance, the flywheels and axle rotate. Upon rotation, the cord unwraps from the axle as the lever arm moves away from the seat to allow the knees to extend. Upon full extension, kinetic energy imparted by flywheel rotation reverses footplate movement as the strap rewraps around the axle; at that time during CE2 workouts eccentric torque slowed both the footplate's return motion and flywheel rotation. The level of 
eccentric loading is reliant on the peak flywheel velocity attained by the prior concentric action. Maximal-effort concentric actions offer more eccentric loading than standard weight training devices (Tesch and Berg, 1997). Unlike the CE2 paradigm, for $\mathrm{CO} 2$ and $\mathrm{CO} 4$ workouts users exert no eccentric torque but rather, once full knee extension occurs, allow the lever arm to return to its position at the start of repetitions unimpeded as their feet remain perched atop the footplate.

\section{Procedures}

As part of a repeated measures design, subjects made four laboratory visits. After familiarization, in which they exercised on the ergometer at a submaximal level of effort, they performed three workouts separated by one week. Though regular meals/food does not impact circadian-based [T] (Grigor'iev et al., 1994), subjects were instructed to consume their normal diets on workout days. Workouts occurred between 1300-1700 hours. Per subject, they occurred at the same time of day and began with a pre-exercise lactate measurement whereby $\sim 20 \mu \mathrm{L}$ of fingertip blood was placed on a test strip inserted within a calibrated analyzer (Accutrend; Hawthorne, NY). Prior research notes the analyzer yielded acceptable [BLa'] (Baldari et al., 2009). With specialized kits (Salimetrics; State College, $\mathrm{PA})$ we obtained saliva to measure [T] due its minimal invasiveness and data reliability in both men and women (Liening et al., 2012; Strollo et al., 2005). Subjects passively drooled for two minutes onto a sublingual swab, which was then immediately inserted into a container and submerged in ice for temporary storage. After a five-minute stationary cycling (Ergotest 812A; Stockholm, Sweden) warm-up, subjects performed a workout. Once a workout was performed, they did not repeat it.

A rotary encoder was mounted on the ergometer to record flywheel velocities at $10 \mathrm{~Hz}$. That data was fed into a computer equipped with software (Model 8.1, Labview, Austin, TX). Work was calculated as: $\mathrm{E}_{\mathrm{kin}}=\mathrm{I} 1 / 2\left(\omega^{2}\right)$ whereby I equals flywheel inertia and $\omega$ denotes the peak velocity per repetition. That formula calculated the work done per repetition and was summed to derive the total work (TW) per exercise bout. Such instrumentation methods have produced acceptable levels of reproducibility (Caruso et al., 2006). For workouts, subjects were instructed to exert maximal effort, received verbal encouragement, and rested 90 seconds between sets. [BLa'] were measured at zero-, five-, ten-, 15-, and 20-minutes post-exercise. Since [BLa $\left.{ }^{-}\right]$ undergo rapid changes in response to exercise, we chose multiple measurements to assess temporal changes to the metabolite. Post-exercise saliva collection occurred (mean \pm sem) $11.6 \pm 0.6$ minutes after the final set per workout. Our salivary assay procedures to quantify [T] were identical to methods used previously by members of our investigative team (Caruso et al., 2012).

\section{Statistics}

We used Z-scores to locate data outliers. Our data was examined for compliance to ANOVA assumptions (normality, independence, equal variances). TW data were compared with a oneway ANOVA; no inter-gender comparison occurred as prior research affirmed higher male TW values (Caruso et al., 2010). [BLa'] were assessed with a 2 (gender) x 3 (workout) x6 (time) ANOVA, with repeated measures for workout and time. [T] were compared with a 2 (gender) $\mathrm{x} 2$ (time) x3 (workout) ANOVA, with repeated measures for workout and time. We used subjects' pre-exercise [T] as a covariate to limit the variability of data provided by both men and women. Scheffe's post-hoc located the source of the differences. An $\alpha=0.05$ was used for all analyses.

\section{RESULTS}

Z-score analyses revealed no outliers and ANOVA assumptions were met. TW data appear in Table 1 and were partitioned into concentric, eccentric, and total values. Table 1 results include a significant $(\mathrm{CE} 2, \mathrm{CO} 4>\mathrm{CO} 2)$ inter-workout difference. [BLa'] appear in Figure 2 and exhibit a workout $\mathrm{x}$ time interaction. The post-hoc revealed differences in [BLa'] per post-exercise time point, whereby $\mathrm{CO} 4$ workouts evoked the highest $\left[\mathrm{BLa}^{-}\right]$ and significantly more than the $\mathrm{CE} 2$ and $\mathrm{CO} 2$ bouts. Only at five-minutes post-exercise did CE2 and $\mathrm{CO} 2\left[\mathrm{BLa}^{-}\right]$differ significantly. Figure 3 displays [T] with gender (men $>$ women) and time (pre $<$ post) main effects. 
Table 1. Work (mean \pm sem, in joules) results.

\begin{tabular}{|c|c|c|c|}
\hline & Concentric & Eccentric & Total (TW) \\
\hline $\mathrm{CO} 2$ & $5074 \pm 275$ & - & $5074 \pm 275$ \\
\hline $\mathrm{CE} 2$ & $4935 \pm 261$ & $4893 \pm 261$ & $9846 \pm 189 *$ \\
\hline $\mathrm{CO} 4$ & $9620 \pm 410$ & - & $9620 \pm 410^{*}$ \\
\hline
\end{tabular}

*: $\mathrm{CE} 2, \mathrm{CO} 4>\mathrm{CO} 2(\mathrm{P}<0.05)$

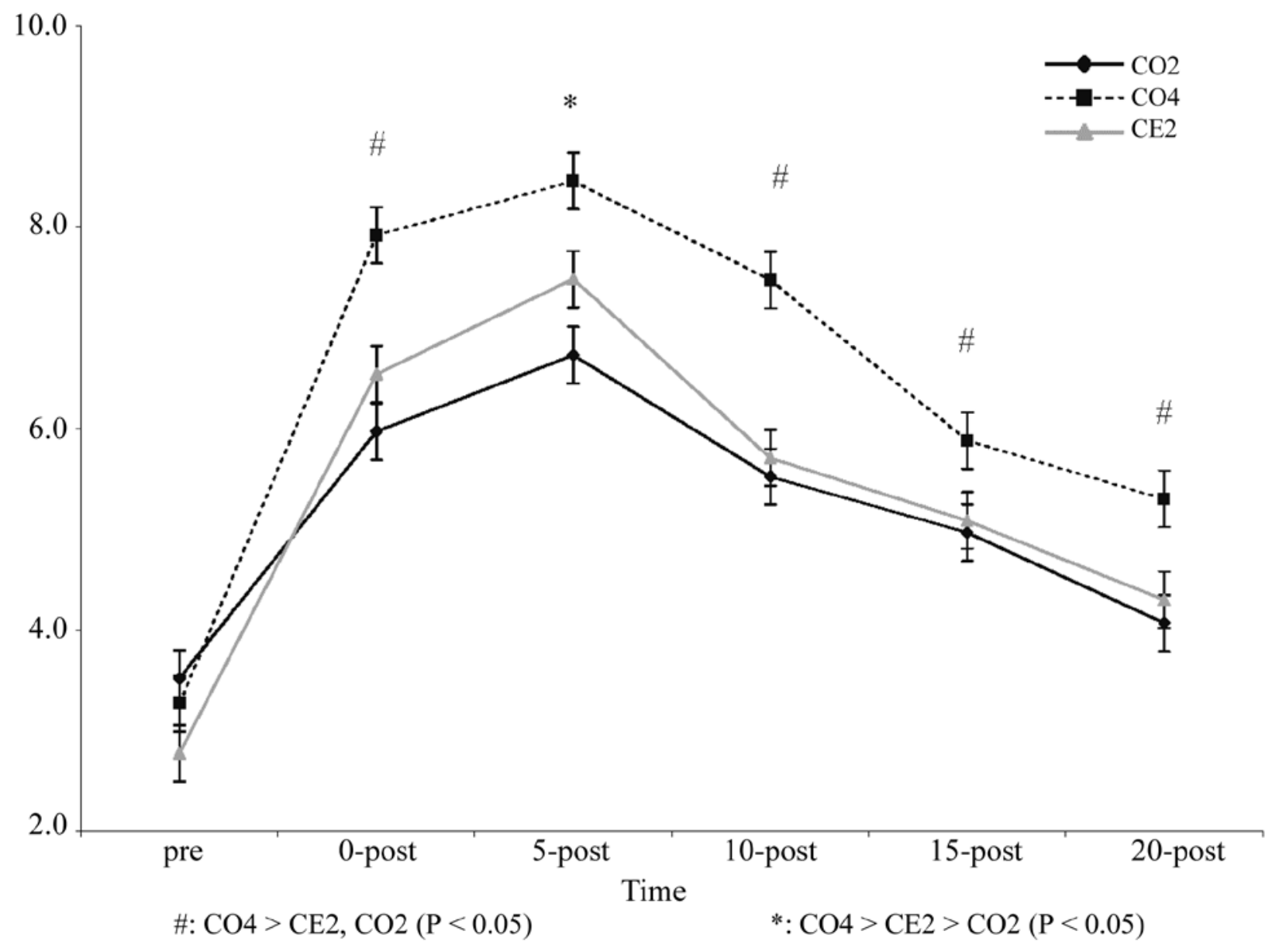

Figure 2. Current study [BLa] over time. Error bars denote sem values. 


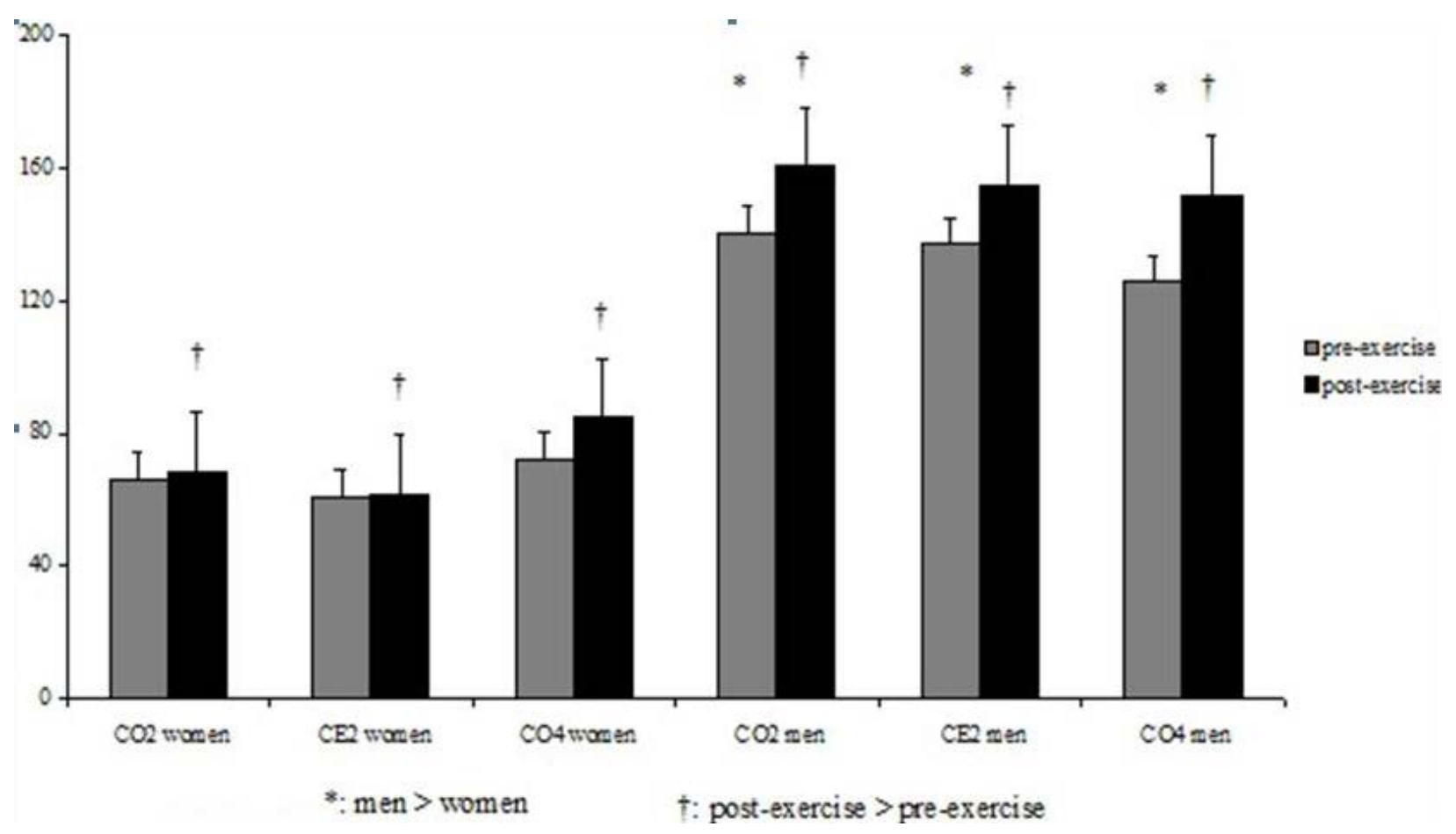

Figure 3. $[\mathrm{T}]\left(\right.$ mean $\pm \mathrm{sem}$, in $\left.\mathrm{pg} \cdot \mathrm{ml}^{-1}\right)$.

\section{DISCUSSION}

Our rationale for a $1-g$ trial to assess changes to $\left[\mathrm{BLa}^{-}\right]$is from research that noted exercise in simulated $\mu g$ does not raise levels of the metabolite beyond those seen from 1-g workouts done at similar intensities (Zamparo et al., 1992). Yet such relationships may not extend to high intensity workouts or during parabolic flights, as the latter may not completely be representative of a spacecraft environment. Despite a similar TW value to the $\mathrm{CO} 4$ exercise bout, the eccentric actions that comprised the CE2 workout did little to raise $\left[\mathrm{BLa}^{-}\right]$beyond those for $\mathrm{CO} 2$. Yet our study noted no inter-workout differences in [T]. Taken together, our data imply large volumes of concentric activity on the prototype ergometer are counterproductive as it did not elicit a significantly greater increase in $[\mathrm{T}]$ than workouts with half the muscle shortening activity. Our results may aid in-flight countermeasure prescriptions, which has relied on exercise with large volumes of concentric activity, such as stationary cycle ergometry (Friel and Vance, 2013). Our hypothesis was affirmed and results may aid exercise prescriptions for flywheel-based hardware.

Concentric actions require a steady supply of ATP to break crossbridges that cause sarcomeres to shorten (Komi et al., 1987). Yet for eccentric exercise, crossbridges break from the external loads imposed upon muscles. Prior studies that compared concentric and eccentric exercise saw stark contrasts between muscle shortening and lengthening actions that may explain the postexercise differences to our [BLa $\left.{ }^{-}\right]$. Weight training done against the same absolute load noted eccentric actions required only $14-16 \%$ of the energy cost of concentric exercise (Dudley et al., 1991; Menard et al., 1991), and even when matched for work muscle lengthening elicited only $20 \%$ of the energy cost of concentric actions (Komi et al., 1987). Our results concur with prior outcomes that suggest eccentric actions require less ATP and yield smaller increases in [BLa $\left.{ }^{-}\right]$ than concentric exercise (Dudley et al., 1991; Komi et al., 1987; Menard et al., 1991).

Our CO4 workout produced greater postexercise [BLa'], presumably since CE2 bout relies less upon ATP to break crossbridges (Komi et al., 
1987). Recent research, with the same ergometer used in our study, assessed the impact of contractile mode and work volume on changes to [BLa'] from three different leg press bouts (Caruso et al., 2010). [BLa $\left.{ }^{-}\right]$were measured before and at five minutes post-exercise and led to a time main effect (post $>$ pre) but no interworkout differences. [BLa] were like those produced on standard weight training equipment and were indicative of the type of protocol performed (Caruso et al., 2010). Discrepancies in post-exercise $\left[\mathrm{BLa}^{-}\right]$results among the current and prior studies may be due to differences in the three workouts used for each investigation. The two-way $\left[\mathrm{BLa}^{-}\right]$interaction seen in the current, but not prior, trial is likely due to our larger subject sample.

Unlike our [BLa'] results, data for our [T] did not include inter-workout differences. Prior research shows [T] deficits in men who bed rested for 27 days as they concurrently exercised (Wade et al., 2005). Subjects were divided into three with no crossover; one group served as sedentary bed rest controls, while the two other bed rest groups concurrently performed supine aerobic or resistive exercise five times per week. Results showed those who exercised experienced significant deficits in [T]; the degree of loss was not significantly different among the two exercise modes. It was noted other factors, such as lower caloric intake and higher energy costs, may interact with exercise to exacerbate losses in [T]. Yet unlike our study, bed rest subjects who received the resistive exercise treatment performed repetitions that isolated movement to the knee joint (Wade et al., 2005). Perhaps highintensity multi-joint exercises, like the current workouts, are best suited to elicit increases in [T] as they engage a greater amount of muscle mass (Bosco et al., 1996a; Bosco et al., 1996b; Ratames et al., 2005).

Intense exercise is linked to endogenous testosterone release and concurs with the time main effect seen in Figure 3. (Bosco et al., 1996a; Bosco et al., 1996b; Ratames et al., 2005). Two lower body resistive exercise protocols were compared for changes in [T] (Ratames et al., 2005). In a randomized order, subjects performed a single- and six-set squat workout. Results included significant increases in [T] for the six-, but not the single-set, squat workout. It was concluded the volume of exercise from the singleset workout was insufficient to raise testosterone levels over time (Ratames et al., 2005). Perhaps differences in inter-workout results for [T] between the prior (Ratames et al., 2005) and current studies are due to the relative similarity of the exercise bouts examined in our trial. Other high-intensity exercise paradigms saw increases in [T]. A 60-second vertical jump test significantly raised free and total [T] by 13 and $12 \%$ respectively (Bosco et al., 1996a). Serum [T] were also significantly correlated to vertical jump and sprint performance (Bosco et al., 1996b). Thus our main effect for time concur with results (Bosco et al., 1996a; Bosco et al., 1996b; Ratames et al., 2005) that saw similar $[\mathrm{T}]$ increases in response to high-intensity multi-joint physical activity paradigms.

Current study exercise bouts produced an approximate $5 \%$ increase in $[\mathrm{T}]$ regardless of the leg press workout examined. Such increases are very comparable to monthly $125 \mathrm{mg}$ injections of long-acting testosterone enanthate given to healthy young men (Bhasin et al., 2001). Concurrent to injections that ceased endogenous secretion of the hormone so that subjects were completely reliant on external sources, testosterone was administered as muscle mass and strength changes were recorded. After 16 weeks, during which they refrained from resistance exercise and moderate-to-heavy aerobic activity, their [T] increased by approximately 5\%. Such increases also evoked significant gains in fat free mass and quadriceps volume (Bhasin et al., 2001).

Acute responses to each of our workouts led to similar [T] elevations. Since such 5\% increases over time led to muscle mass gains (Bhasin et al., 2001) and injectable testosterone is associated with health risks, frequent bouts of high-intensity in-flight FWED workouts may be a more prudent choice to reduce muscle mass and strength losses. However, since exercise leads to rapid but only temporary increases in [T] (Liu et al., 2009), the frequency of flywheel-based workouts in $\mu \mathrm{g}$ should be higher than when similar protocols are done in 1-g (Rantalainen and Klodowski, 2011; Umemura et al., 2002). Such exercise bouts would inevitably entail application of high muscle/reaction forces that characterize resistive exercise done in 1-g and may therefore best abate muscle mass and strength losses incurred during 
$\mu g$ (Rantalainen and Klodowski, 2011). Due to its level of importance, future research should assess testosterone and lactate changes from flywheelbased resistive exercise hardware in simulated $\mu g$, as well as continued inquiry into flywheel-based exercise prescriptions (NASA Human Research Roadmap, 2014).

\section{REFERENCES}

Baldari C, Bonavolontà V, Emerenziani GP, Gallotta MC, Silva AJ, Guidetti L (2009) Accuracy, reliability, linearity of Accutrend and Lactate Pro versus EBIO plus analyzer. European Journal of Applied Physiology 107: 105-111

Bentley JR, Leach MA, McCleary F, Smith C, Norcross J, Hagan RD, Dao A, Zamaitis J, Landeck M, Groat M, Carrejo D, Cooper J, Weisskopf G, Mangieri M (2006) Advanced Resistive Exercise Device (ARED) Man-InThe-Loop Test (MILT). NASA Technical Memorandum 2006-213717

Bhasin S, Woodhouse L, Casaburi R, Singh AB, Bhasin D, Berman N, Chen X, Yarasheski K, Magliano L, Dzekov C, Dzekov J, Bross R, Phillips J, Sinha-Hikim I, Shen R, Storer T (2001) Testosterone dose-response relationships in healthy young men. American Journal of Physiology 281: E1172-E1181

Bosco C, Tihanyl J, Rivalta L, Parlato G, Tranquilli C, Pulvirenti G, Foti C, Viru M, Viru A (1996a) Hormonal responses in strenuous jumping effort. Japanese Journal of Physiology 46: 93-98

Bosco C, Tihanyi J, Viru A (1996b) Relationships between field fitness test and basal serum testosterone and cortisol levels in soccer players. Clinical Physiology 16: 317-322

Caruso JF, Coday MA, Monda JK, Ramey ES, Hastings LP, Vingren JL, Potter WT, Kraemer WJ, Wickel EE (2010) Blood lactate and hormonal responses to prototype flywheel ergometer workouts. Journal of Strength and Conditioning Research 24: 749-756

Caruso JF, Lutz BM, Davidson ME, Wilson K, Crane C, Craig C, Nisson T, Mason ML, Coday MA, Sheaff R, Potter WT (2012) Salivary hormonal values from high-speed resistive exercise workouts. Journal of
Strength and Conditioning Research 26: 625632

Caruso JF, Williams JA, Hari P, McCoy JD, Coday MA, Ramsey CA, Capps LB (2006) Data reproducibility from the instrumentation of an inertial resistance exercise device suggested for use during space travel. Isokinetics and Exercise Science 14: 371-382

Dudley GA, Tesch PA, Harris RT, Golden CL, Buchanan P (1991) Influence of eccentric actions on the metabolic cost of resistance exercise. Aviation, Space, and Environmental Medicine 62: 678-682

European Space Agency (2009) ESA ISS Science \& System-Operations Status Report Increment 21. Retrieved from http://www. esa.int/Our_Activities/Human_Spaceflight/Co lumbus/ESA_ISS_Science_System_-_Opera tions Status Report br Increment 212

Friel J, Vance J (2013) Triathlon Science. Champaign, IL: Human Kinetics

Garnier J (2012) Stanford software, NASA help launch eighth-grade science project. Retrieved from http://simbios.stanford.edu/ PressReleases/2012apr opensim.htm

Grigor'iev AI, Noskov VB, Popova IA, Vetrova EG, Markin AA, Strogonova LB (1994) Effects of a long-term space flight on human biochemical status. Klinical Laboratory Diagnostics 1: 19-22

International Countermeasures Working Group (2010) International Exercise Countermeasures Hardware Catalog. Retrieved from http://www.nasa.gov/centers/johnson/pdf/505 724main_International_Exercise_Countermea sures_Hardware_Catalog.pdf

Komi PV, Kaneko M, Aura O (1987) EMG activity of the leg extensor muscles with special reference to mechanical efficiency in concentric and eccentric exercise. International Journal of Sports Medicine 8: 22-29

Kraemer WJ, Gordon SE, Fleck SJ, Marchitelli L, Mello R, Dziados JE, Friedl K, Harman E, Maresh C, Fry AC (1991) Endogenous anabolic hormonal and growth factor responses to heavy resistance exercise in males and females. International Journal of Sports Medicine 12: 228-235

Liening SH, Stanton SJ, Saini EK, Schultheiss OC (2012) Salivary testosterone, cortisol, and 
progesterone: Two-week stability, interhormone correlations, and effects of time of day, menstrual cycle, and oral contraceptive use on steroid hormone levels. Physiology and Behavior 99: 8-16

Liu TC, Kuo CH, Wang PS (2009) Exercise and testosterone. Adaptive Medicine 1: 26-31

Menard MR, Penn AM, Lee JWK, Dusik LA, Hall LD (1991) Relative metabolic efficiency of concentric and eccentric exercise determined by 31P magnetic resonance spectroscopy. Archives of Physical Medicine and Rehabilitation 72: 976-983

NASA (2014) Human Research Roadmap (formerly NASA Critical Path Roadmap in 2000). Retrieved from http://human researchroadmap.nasa.gov/

Peterman MM, Hamel AJ, Cavanagh PR, Piazza SJ, Sharkey NA (2001) In vitro modeling of human tibial strains during exercise in microgravity. Journal of Biomechanics 34: 693-698

Rantalainen T, Klodowski A (2011) Estimating lower limb skeletal loading. In Theoretical Biomechanics, V. Klika (eds), pp 243-266. Kroatia: InTech

Ratames NA, Kraemer WJ, Volek JS, Maresh CM, VanHeest JL, Sharman MJ, Rubin MR, French DN, Vescovi JD, Silvestre R, Hatfield DL, Fleck SJ, Deschenes MR (2005) Androgen receptor content following heavy resistance exercise in men. Journal of Steroids and Biochemistry in Molecular Biology 93: $35-42$

Stauber WT (1989) A unique problem of muscle adaptation from weightlessness: the deceleration deficiency. In NASA Technical Report, Workshop on Exercise Prescription for Long-Duration Space Flight, B.A. Jr. Harris, D.F. Stewart (eds), pp 57-59. Washington, DC: NASA

Strollo F, Boitani C, Basciani S, Pecorelli L, Palumbo D, Borgia L, Masini MA, Moré M, Strollo G, Spera G, Uva BM, Riondino G (2005) The pituitary-testicular axis in microgravity: analogies with the ageing male syndrome. Journal of Endocrinological Investigations 28: S78-S83

Tesch PA, Berg HE (1997) Resistance training in space. International Journal of Sports Medicine 18: S322-S324

Umemura Y, Sogo N, Honda A (2002) Effects of intervals between jumps or bouts on osteogenic response to loading. Journal of Applied Physiology 93: 1345-1348

Wade CE, Stanford KI, Stein TP, Greenleaf JE (2005) Intensive exercise training suppresses testosterone during bed rest. Journal of Applied Physiology 99: 59-63

Zamparo P, Capelli C, Antonitto G (1992) Blood lactate during leg exercise in microgravity. Acta Astronautica 27: 61-64 